\title{
Improved wavelet reassigned scalograms and application for modal parameter estimation
}

\author{
Z. K. Peng ${ }^{\mathrm{a}}$, G. Meng ${ }^{\mathrm{a}}$ and F. L. Chu ${ }^{\mathrm{b}, *}$ \\ ${ }^{a}$ State Key Laboratory of Mechanical System and Vibration, Shanghai Jiao Tong University, Shanghai, 200240, \\ P.R. China \\ ${ }^{\mathrm{b}}$ Department of Precision Instruments, Tsinghua University, Beijing 100084, P. R. China
}

Received 5 February 2010

Revised 4 May 2010

\begin{abstract}
The present work carries out a comprehensive investigation into the border distortion deficiency for the conventional scalogram and the reassigned scalogram. The reasons for this deficiency are analyzed, and a simple way is suggested to determine the border distortion ranges. New methods are proposed to reduce the border distortions in both the scalograms. The practical meanings of the border distortion improvement method are demonstrated by applying both the scalograms with and without border distortion improvements to estimate the modal parameters for a 2-DOF linear system. The estimation results indicate that, in the presence of noise, which is inevitable in practice, for the mode with weak amplitude, the reassigned scalogram can perform better than the conventional scalogram in estimating the modal parameters. In addition, for the mode with short effective duration, its frequency components at the border distortion ranges are of great importance for the modal parameter estimation purpose, and the border distortion improvement method can greatly increase the estimation accuracy.
\end{abstract}

Keywords: Wavelet transform, border distortion, edge effect, modal analysis, scalogram, reassigned scalogram

\section{Introduction}

Over the past twenty years, wavelet theory [1] has become one of the emerging and fast-evolving mathematical and signal processing tools for its many distinct merits. Different from the traditional Fourier Transform which is generally believed to be less competent in the non-stationary signal analysis [2], by introducing the dilation and translation operators the Wavelet Transform (WT) can achieve a multi-scale analysis for a signal and, therefore, it can effectively extract time-frequency features of both the stationary signals and the non-stationary signals [2]. In the field of mechanical signal processing, the WT has been widely used for the singularity detection [3-6], the noise reduction [7-9], and the feature extraction [10-13] and et al. Especially, the wavelet scalogram [14], which is usually regarded as the counterpart of the spectrum of the Short Time Fourier Transform (STFT) [15], has been widely adopted for analyzing the non-stationary vibration signals and extracting time-frequency features from vibration signals for the fault diagnosis purpose [16-19]. In addition, the ridge and skeleton of wavelet transform have shown excellent capability in tracing the instantaneous characteristics of the system dynamic responses such as the timedependent amplitude and the amplitude-dependent frequency of the free response of both the linear and nonlinear systems. This distinct capability allows the WT method to play important roles in the parameter identifications for both linear and nonlinear systems [20-33]. A comprehensive review about the application of the WT method in mechanical signal analysis can be found in Ref. [34].

\footnotetext{
${ }^{*}$ Corresponding author. E-mail: chufl@mail.tsinghua.edu.cn.
} 
While praising the powerful capabilities of the WT, enjoying the benefits from the WT and being encouraged for the achievements provided by the WT, it is better for us to be aware that the WT will also suffer from some deficiencies such as the limitation defined by the Heisenberg-Gabor inequality [35], the frequency band overlapping and interference $[36,37]$ and the border distortion [24-28] also known as edge effect which is usually caused by the insufficient data points both at the beginning and at the end of finite-duration signals. Compared to the other two deficiencies, the border distortion deficiency has received relatively more attentions from researchers, especially who are interested in applying the WT method for system identification studies because the border distortion can make significant effects on the identification results. To alleviate effects of the border distortion, signal padding based methods have been mainly applied such as the zero padding and symmetrical padding. Actually, there is a close relationship between the first two deficiencies. The Heisenberg-Gabor inequality makes it impossible for the WT to obtain both fine resolutions in time and in frequency at the same window defined in the time-frequency plane. Moreover, due to the deficiency of frequency band overlapping, the interference may occur in the time-frequency plane. Therefore, the results generated by the wavelet scalograms might be somewhat difficult to be interpreted by researchers and participators. To improve the readability of the wavelet scalograms, Auger and Flandrin [35] have put forward a reassignment method, which works by shifting the components from the geometrical centre of the resolution window of the time-scale analysis to the gravity centre of their complex energy density distribution. After the reassignment processing, the scalogram will have better time and frequency concentration and less interference. The energy density contained in the components of the reassigned scalogram will be closer to that of the original inspected signal. Therefore, the reassignment method can to a certain degree overcome the limitation defined by the Heisenberg-Gabor inequality and reduce the frequency band overlapping and interference in the time-frequency plane. However, it is a pity that the reassignment method could not alleviate the deficiency of the border distortion, and what is more, the reassignment operation could introduce a new kind of border distortion to the reassigned scalogram which has different forms with the border distortion of the conventional scalogram.

The present research will be devoted to addressing the border distortions for the conventional scalogram and the reassigned scalogram, the causes of which will be investigated. New methods will be put forward to reduce the border distortions for both the conventional and reassigned scalogram and the improved scalograms will then be used to estimate the modal parameters for a MDOF (Multiple Degrees of Freedom) linear system. The applications of the WT in the mechanical signal processing are briefly reviewed and the deficiencies of the WT are mainly explained in this section. Section 2 presents the theory of the continuous WT and a brief introduction of the conventional and reassigned scalograms. Section 3 investigates the border distortions of the scalogram and the reassigned scalogram, and new methods are proposed to tackle these border distortion deficiencies. Section 4 presents the applications of using the improved scalograms for the modal parameter estimation for a MDOF linear system. Finally, section 5 lays out the conclusive remarks and the benefit of this research.

\section{Wavelet transform theory}

The driving force behind the WT is to overcome the disadvantages of the short time Fourier transform (STFT), which provides constant resolution for all frequencies since it uses the same window for the analysis of the inspected signal $x(t)$. On the contrary, The WT can achieve a multi-resolution analysis to the inspected signal. That is they use different window functions to analyze different frequency bands of the signal $x(t)$. The different window functions $\psi_{a, b}(t)$, which are also called son wavelets, can be generated by dilation or compression of a mother wavelet $\psi(t)$. The WT can be categorized as discrete WT and continuous WT.

\subsection{Continuous wavelet transform}

Assume $x(t)$ to be a finite-energy function, that is, $x(t) \in L^{2}(R)$. The continuous wavelet transform of $x(\mathrm{t})$ is defined as the inner product in the Hilbert space of the $L^{2}$ norm as follows,

$$
W_{x}(a, b ; \psi)=<x(t), \psi_{a, b}(t)>=\int x(t) \psi_{a, b}^{*}(t) d t \quad a>0
$$


where the asterisk stands for complex conjugate and the family of wavelet $\psi_{a, b}(t)$ consists of a series of son wavelets, which are generated by dilation and translation from the mother wavelet $\psi(t)$, shown as follows,

$$
\psi_{a, b}(t)=a^{-1 / 2} \psi\left(\frac{t-b}{a}\right)
$$

where $a$ is the scale parameter, $b$ is the time parameter, and both of them vary continuously; the factor $a^{-1 / 2}$ is used to ensure energy preservation for transform. Many functions can be used as the mother wavelet, but it must belong to $L^{2}(R)$ and satisfy the following conditions:

1. The definition domain is compact support, which ensures that the function is fast decaying, and so time localization can be obtained.

2. The admissibility condition

$$
C_{\psi}=\int \frac{|\hat{\psi}(\omega)|^{2}}{|\omega|} d \omega<\infty
$$

where $\hat{\psi}(\omega)=\int \psi(t) e^{-i \omega t} d t$. This condition imposes a band-pass characteristic to the spectrum of the wavelet function and guarantees the existence of a reconstruction formula and makes that the waveform of the mother wavelet function must be oscillating.

Wavelet transform is a linear transform, whose physical pattern is to use a series of oscillating functions with different frequencies as window functions $\psi_{a, b}(t)$ to scan and translate the signal of $x(t)$, where $a$ is the dilation parameter for changing the oscillating frequency. Although the wavelet transform is similar to the STFT to a certain sense, the differences between them exist. Compared with the STFT, whose time-frequency resolution is constant, the time-frequency resolution of the wavelet transform depends on the frequency of the signal. For the components of high frequency, the wavelet can reach a high time resolution but a low frequency resolution but, on the other hand, for the components of low frequency, the wavelet transform can provide a high frequency resolution and a low time resolution.

In this paper, the Morlet wavelet is adopted. It is a kind of complex wavelet and can extract the amplitude and phase information of the inspected signal, defined as follows

$$
\psi(t)=\left(\sigma^{2} \pi\right)^{-1 / 4} e^{-i \omega_{0} t} e^{-t^{2} /\left(2 \sigma^{2}\right)}
$$

There is a great deal of wavelets that could be used and, however, the Morelet wavelet is particularly attractive for vibration signal analysis. First, the Morlet wavelet function is an exponentially sinusoidal signal and the damped sinusoidal is the common response of many dynamical system. Second, the Morlet wavelet has a single frequency. If a signal correlates highly with a scaled Morlet wavelet, then the frequency of the wavelet indicates the frequency of the inspected signal.

\subsection{Scalogram and reassigned scalogram}

For all $\psi(t), x(t) \in L^{2}(R)$, the inverse wavelet transform of $x(t)$ is defined as

$$
x(t)=\frac{1}{C_{\psi}} \iint a^{-2} W_{x}(a, b ; \psi) \psi_{a, b}(t) d a d b
$$

It can be known from Eq. (5) that after the application of WT to the signal, it does not lose any information as the energy is preserved during the transformation. Therefore, Eq. (5) is tenable as

$$
<x(t), x(t)>=\int|x(t)|^{2} d t=\frac{1}{C_{\psi}} \int a^{-2} d a \int\left|W_{x}(a, b ; \psi)\right|^{2} d b
$$

A new item $S G_{x}$ can be defined from Eq. (6) as $\left|W_{x}(a, b ; \psi)\right|^{2}$, which is named as the wavelet scalogram [14]. The scalogram shows how the energy of the signal varies with the time and the frequency. It has been widely used for analyzing non-stationary signals. The scalogram can be seen as a spectrum with constant relative bandwidth. It could be easier for people to understand the practical meaning of the scalogram if regarding the scalogram as 


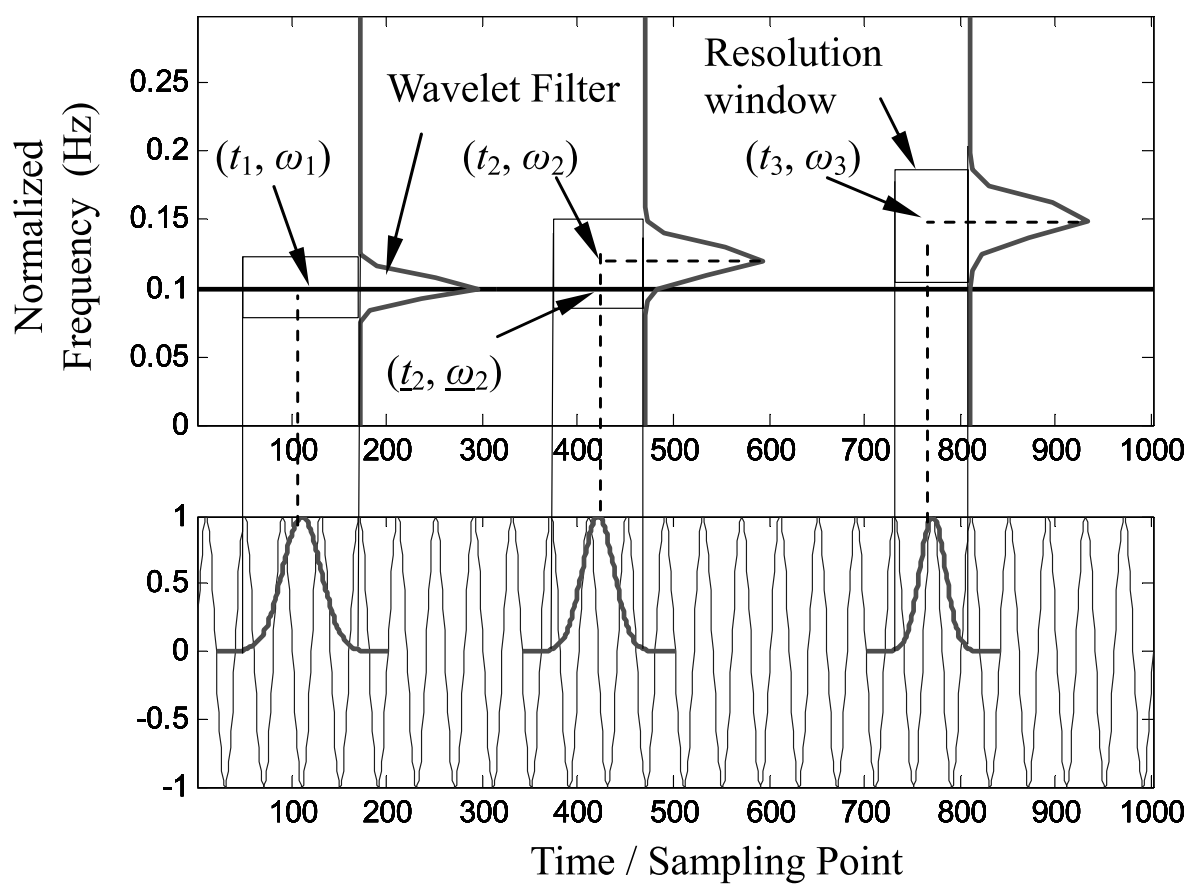

Fig. 1. A sinusoidal signal and the illustration of the wavelet transform.

the outcome of scanning the signal at very point using a series of band-pass filters with different central frequency and compact time support, and a big central frequency corresponding to a big frequency bandwidth and a short time support and vice versa. Moreover, when the Morlet wavelet function is employed, the central frequency of the band-pass filter at scale $a$ can be determined as follows,

$$
\omega=\omega_{0} / a
$$

The operation of the WT as band-pass filters can be well demonstrated by the example signal shown in Fig. 1 where the inspected signal is a sinusoidal signal whose frequency is assumed to be normalized at $0.1 \mathrm{~Hz}$. It is shown that the value of scalogram at point $\left(t_{1}, \omega_{1}\right)$ is actually generated by picking up the signal fraction around $t_{1}$ with a time window determined by the wavelet function and then passing them into the band-pass filter whose central frequency is $\omega_{1}$. Therefore, $\left|W_{x}\left(\omega_{1}, t_{1} ; \psi\right)\right|^{2}$ actually represents the average energy of the signal in the resolution window determined by the rectangle whose geometrical center is $\left(t_{1}, \omega_{1}\right)$. The generation of the scalogram at points $\left(t_{2}, \omega_{2}\right)$ and $\left(t_{3}, \omega_{3}\right)$ can be explained in the same way. Obviously, the scalogram at point $\left(t_{1}, \omega_{1}\right)$ will have the biggest value as the frequency of the signal fraction is coincident with the central frequency of the wavelet filter and so the energy of the signal fraction can completely pass through the filter. For point $\left(t_{2}, \omega_{2}\right)$, as only fractional energy of the signal can pass through the wavelet filter and, therefore at this point the value of the scalogram is not zero but quite small. Similarly it can be predicted that at point $\left(t_{3}, \omega_{3}\right)$ the value of the scalogram is zero. According to the intuitive understanding, the non-zero scalogram at point $\left(t_{2}, \omega_{2}\right)$ will indicate that the inspected signal contains a component of frequency $\omega_{2}$ at moment $t_{2}$. Clearly, this contradicts the fact there is no frequency component at $\left(\omega_{2}\right)$ in the currently considered signal. The appearance of non-zero scalogram at point $\left(t_{2}, \omega_{2}\right)$ is just caused by the so-called frequency band overlapping deficiency. In the multi-component cases, this deficiency can give rise to interferences in the scalogram. To obtain a scalogram without any frequency band overlapping, it is required that the WT has absolutely fine resolutions both in time and in frequency, which means the area of resolution window must be infinitesimal. However, according to the wavelet theory and the Heisenberg-Gabor inequality, the area of the resolution windows is a constant which may vary with the type of the used wavelet function but can never be close to zero. Therefore, the frequency band overlapping is inevitable, and the interferences caused by the frequency band overlapping can significantly reduce the readability of the scalogram. 
To improve the readability of conventional scalograms, Auger and Flandrin [35] has continued the research of Kodera et al. [38], who had developed the reassignment method for the STFT. Auger and Flandrin reapplied the reassignment method to WT scalogram for better time-frequency representations of the inspected signal. The reassignment method works by shifting the components from the geometrical centre of the resolution window to the gravity centre of the complex energy density distribution. For the sinusoidal signal presented in Fig. 1, obviously the geometrical centre of the resolution window around point $\left(t_{1}, \omega_{1}\right)$ coincides with the gravity centre of the energy distribution of the signal fraction in the resolution window and, therefore, no shift will happen to this point. In addition, as there is no energy distributed in the resolution window around $\left(t_{3}, \omega_{3}\right)$, no shift will happen to this point as well. However, for point $\left(t_{2}, \omega_{2}\right)$, clearly, the gravity centre of the energy distribution of the signal fraction in the resolution window is $\left(\underline{t_{2}}, \omega_{2}\right)$ which is different from the geometrical centre $\left(\underline{t_{2}}, \omega_{2}\right)$. By the reassignment rule, the value at $\left(t_{2}, \omega_{2}\right)$ will be assigned to point $\left(\underline{t}_{2}, \underline{\omega}_{2}\right)$, and the value at $\left(t_{2}, \omega_{2}\right)$ is set to zero. It is worthy nothing here that, in this case, the energy distribution in the resolution window around $\left(t_{2}, \omega_{2}\right)$ is symmetric about $t_{2}$ and thus $t_{2}=\underline{t}_{2}$ and the shift happens only in the frequency direction but, for the complicated signals, it is quite possible for $t_{2} \neq \underline{t}_{2}$ and so the shift would also happen in the time direction. Obviously, after the reassignment, the energy density distribution represented by the reassigned scalogram is closer to that of the original inspected signal, and so the reassigned scalogram will have better time and frequency concentration and less interference. Because of the better concentration and less interference, components that contain low energy could be possibly identified in the reassigned scalogram. Due to the merits of the reassigned scalogram, it has been successfully applied to detect the occurrence time of the rub-impact between the surface of a rotor and the surface of a stator [39] and extract the signature for mechanical watch movements [40].

Detailed information on reassigned scalogram and its algorithm can be found in Ref [35]. The theories of reassigned scalogram can be briefly defined as:

$$
R S G_{x}(\hat{a}, \hat{b} ; \psi)=\iint(\hat{a} / a)^{2} S G_{x}(a, b ; \psi) \delta\left(\hat{b}-b^{\prime}(a, b)\right) \delta\left(\hat{a}-a^{\prime}(a, b)\right) d a d b,
$$

where

$$
\begin{aligned}
& b^{\prime}(a, b)=b-\operatorname{Re}\left\{a \frac{W_{x}\left(a, b ; \psi^{\prime}\right) W_{x}^{*}(a, b ; \psi)}{\left|W_{x}(a, b ; \psi)\right|^{2}}\right\} \\
& \frac{\omega_{0}}{a^{\prime}(a, b)}=\frac{\omega_{0}}{a}+\operatorname{Im}\left\{\frac{W_{x}(a, b ; \hat{\psi}) W_{x}^{*}(a, b ; \psi)}{2 \pi a\left|W_{x}(a . b ; \psi)\right|^{2}}\right\}, \text { and } \\
& \psi^{\prime}(t)=t \psi(t), \hat{\psi}(t)=\frac{d \psi}{d t}(t) .
\end{aligned}
$$

The conventional scalogram and the reassigned scalogram of the signal shown in Fig. 1 are presented in Figs 2 and 3 respectively. Clearly, the reassigned scalogram has better concentration than the conventional scalogram.

\section{Border distortions of the wavelet transform}

The border distortions of the wavelet transform are no longer strange to people now, which is usually caused by the insufficient data points at both the beginning and the end of the finite-duration signal [41,42]. Various methods have been put forward to alleviate the border distortion. In this section, the cause of the border distortion will be explained for both the conventional scalogram and the reassigned scalogram from the band-pass filter point of view and new methods will proposed to eliminate the undesired effect. 


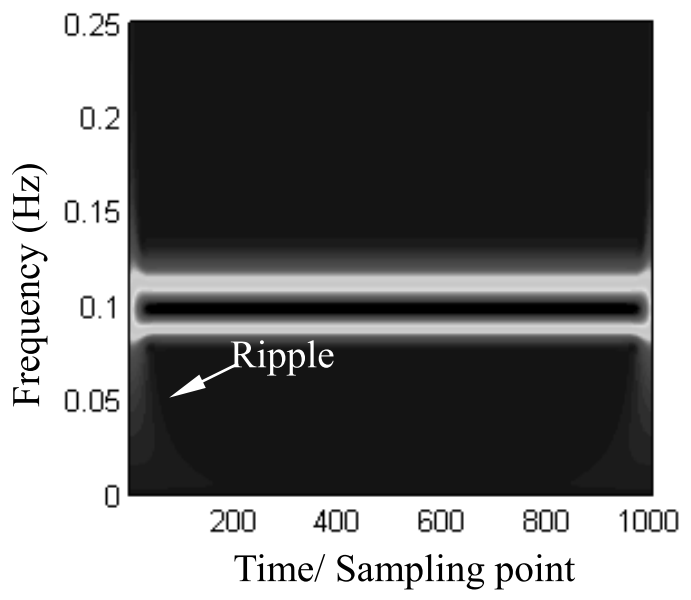

Fig. 2. The conventional scalogram.

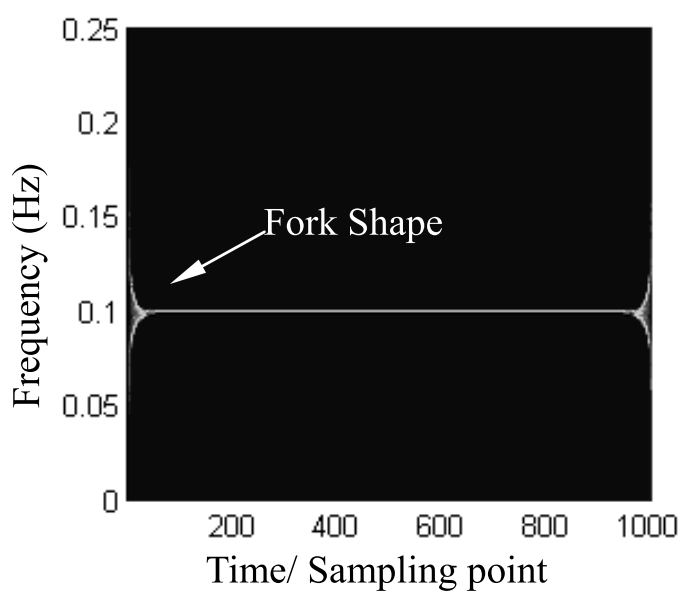

Fig. 3. The reassigned scalogram.

\subsection{The causes of border distortion}

A well-known phenomenon of the border distortion is the energy of the inspected signal will be scattered at both ends. This can be easily explained by linking the wavelet functions to band pass filters. As aforementioned in Section 2, it is known that only the functions that can satisfy the admissibility condition can be used wavelet functions. This implies that the spectrum of the wavelet function must be of band-pass characteristic. A complete wavelet function is of course satisfy the admissibility condition but, however, the wavelet transform at the two edges of the inspected signal is actually like using incomplete wavelet functions to execute the transforms. The incomplete wavelet functions no longer satisfy the admissibility condition and their spectra then are not of strict band-pass characteristics. An illustration to this is shown in Fig. 4. Obviously, for the incomplete wavelet function, the spectrum is not strictly band-pass and its bandwidth is wider than that of the complete wavelet function. Moreover, when using the incomplete wavelet function to pick up the signal fraction, it can also cause spectrum variation and, as shown in Fig. 5, some energy is scattered and thus the spectrum will usually have a wider frequency band than the spectrum of the signal fraction picked up using a complete wavelet function. This scattered energy can be easily understood by comparing the beginning or end of the incomplete signal fraction to a step component which, as well known, has a wide energy distribution in the frequency domain where the components at low frequency region are generally bigger than those at high frequency region. The spectrum of the incomplete signal fraction 

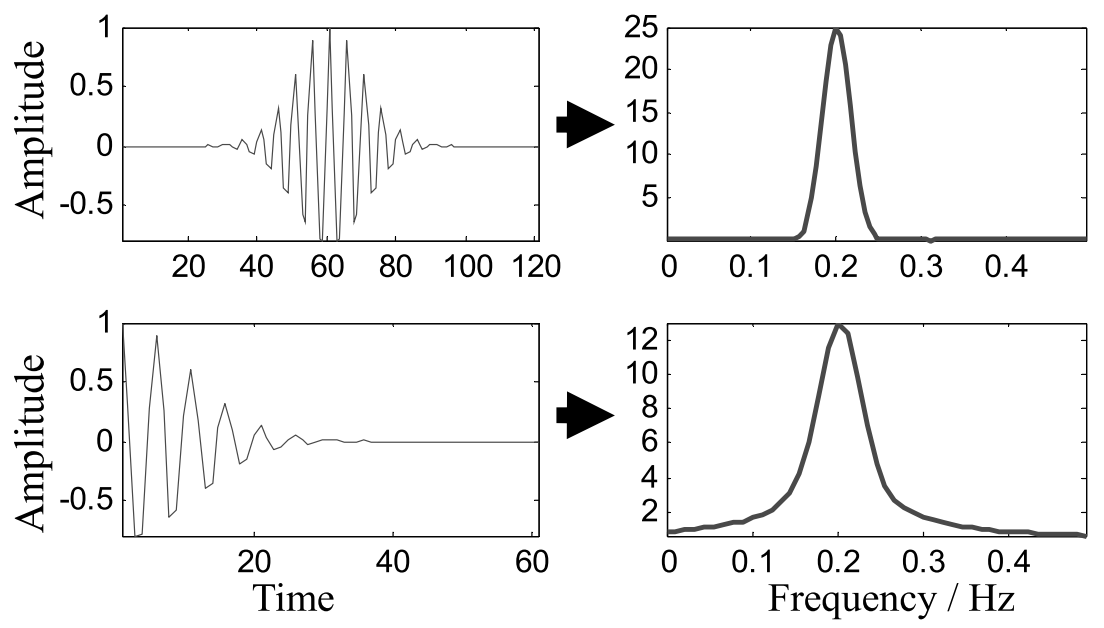

Fig. 4. Complete and incomplete wavelet functions.
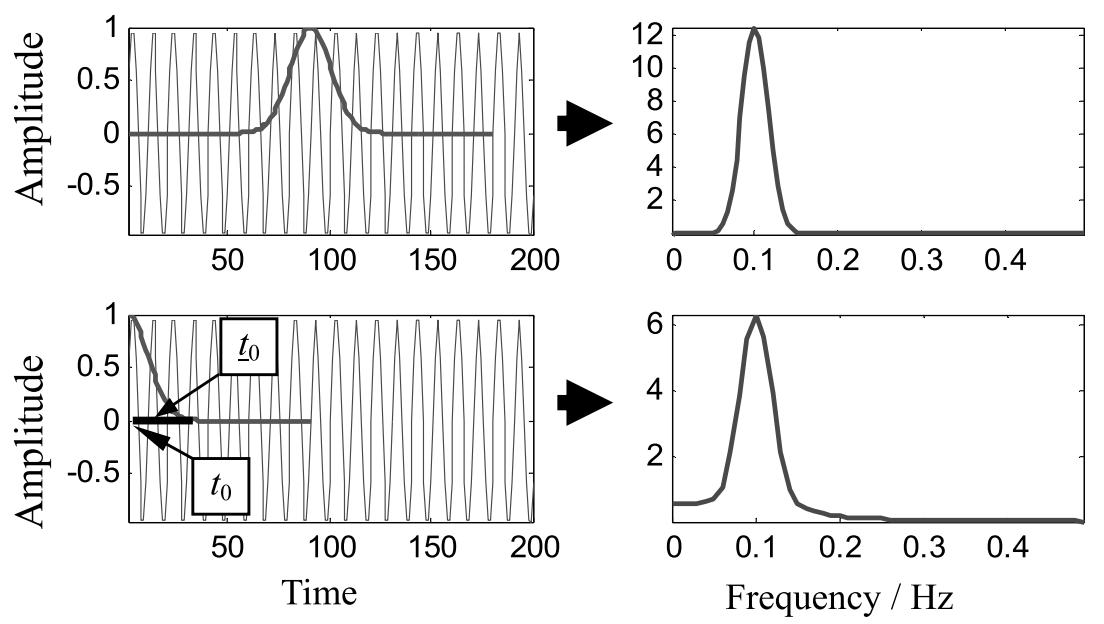

Fig. 5. Signal fractions picked up using complete and incomplete wavelet functions.

then can be regarded as the mixture of the essential frequency components of the signal fraction and the frequency components of the step component. Usually, the former would be much bigger than the latter in amplitude. These two factors working together generate the border distortion. After passing the signals fractions to the two kinds of filters which are determined by the complete and incomplete wavelet functions respectively, the energy distributions in the frequency domain are shown in Fig. 6. Clearly, in the complete function case, the energy is very small and can be ignored and, on the contrary, in the incomplete function case the energy level is relatively significant and a not small value will therefore be assigned to the $0.2 \mathrm{~Hz}$ point even there is actually no energy distribution. The non-zero scalogram at both the edges caused by the energy scattering is also called ripple in Ref [41].

Another typical phenomenon about the border distortion is the amplitudes of the scalogram are generally smaller on the two edges than at the middle part. This is the problem that researchers have often investigated and, aiming at which the methods for border distortion reduction have often been developed, such as the signal padding method. It is easy to identify this phenomenon in scalogram, especially from the values of the scalogram along the ridge. The values of the scalogram ridge in Fig. 2 are shown in Fig. 7, which clearly indicates that the values on the two edges are smaller than at the middle part. It is not difficult to understand this phenomenon. Obviously, the values of the scalogram at the middle part are actually contributed by more signal points than on the two edges and therefore the values of the scalogram at the two edges are usually smaller than at the middle part. 

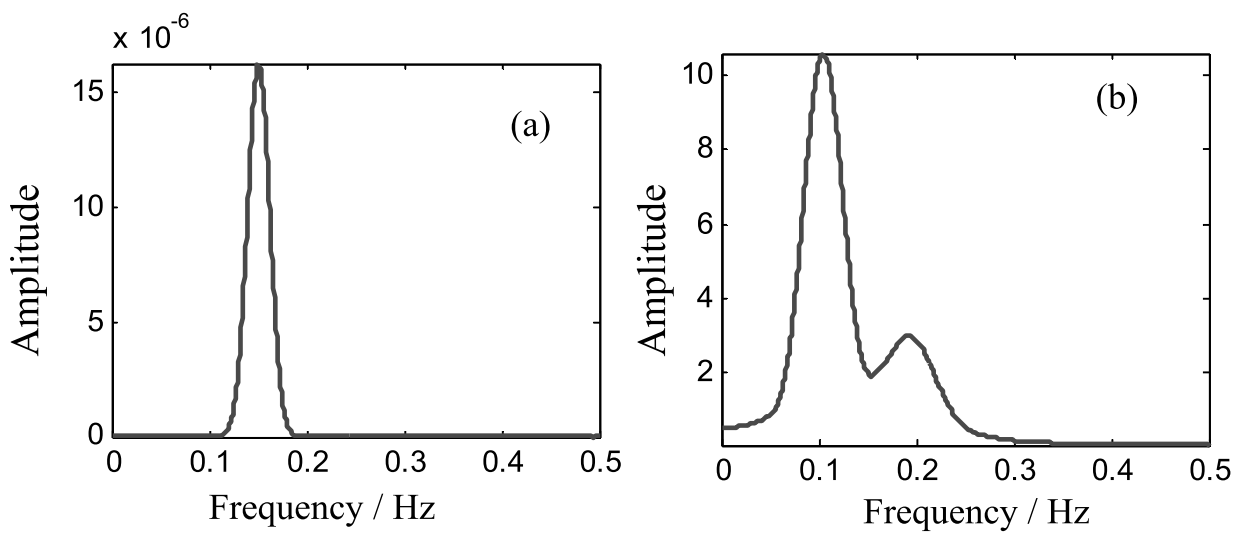

Fig. 6. The energy distributions in the frequency direction [(a)- complete function case; (b)-incomplete function case].

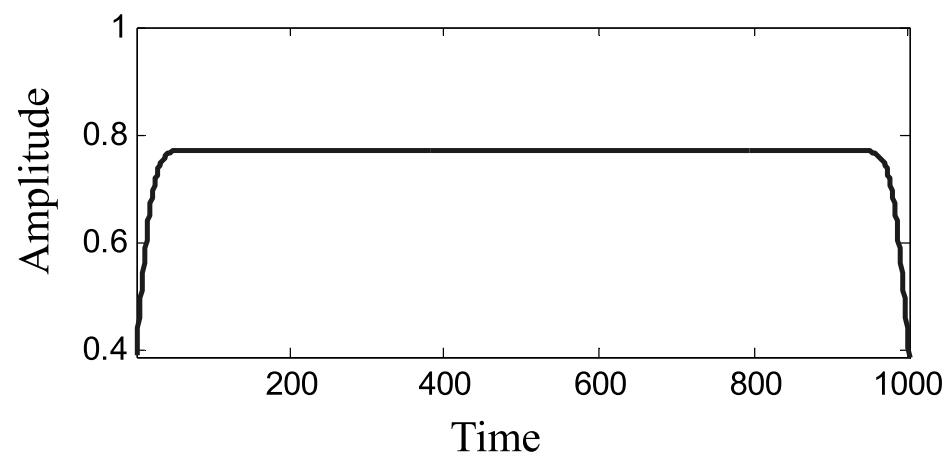

Fig. 7. The values of the scalogram ridge in Fig. 2.

Moreover, it can be seen from Fig. 6(b) that the gravity centre of the energy distribution in the frequency direction will locate between $0.1 \mathrm{~Hz}$ and $02 \mathrm{~Hz}$ and is no longer exactly consistent with that of the inspected signal fraction. Therefore, the reassignment method introduced Section 2.2 can not completely solve the energy scattering problem caused by the border distortion but can still somewhat improve the concentration in the frequency direction. In addition, it can be seen from Fig. 5 that the gravity centre in the time direction for the incomplete wavelet function case will not consistent with the supposed one at $t_{0}$ and will locate somewhat inward at $\underline{t}_{0}$. Therefore, by the reassignment rule, the value at $t_{0}$ will be shifted in time direction to point $\underline{t}_{0}$, and the scalogram value at $t_{0}$ is set to zero. In this way, the reassignment operation will introduce a new kind of border distortion to the reassigned scalogram which has different form with the border distortion of the conventional scalogram. The unique border distortion of the reassigned scalogram is usually of a fork shape, which can be easily observed from Fig. 3 and can also be found from the reassigned scalograms reported by other researchers $[35,40]$.

From the perspective of band pass filter, a comprehensive and profound explanation has been made so far to the appearance of border distortion in the conventional and reassigned scalograms.

\subsection{The effect ranges of border distortion}

As the border distortion can usually introduce negative influences to the applications of the wavelet method in practices, it is then necessary for participators to be aware of the range where the influence of the border distortion can reach in the scalogram and to pay particular attentions to the scalogram at the range. Different methods [41,42] have been suggested to determine the effect range of the border distortion and a general knowledge is the effect range of the border distortion is proportional to the length of the resolution window in time direction. In time direction, the resolution window length of the wavelet function with $a=1$ can be calculated as 


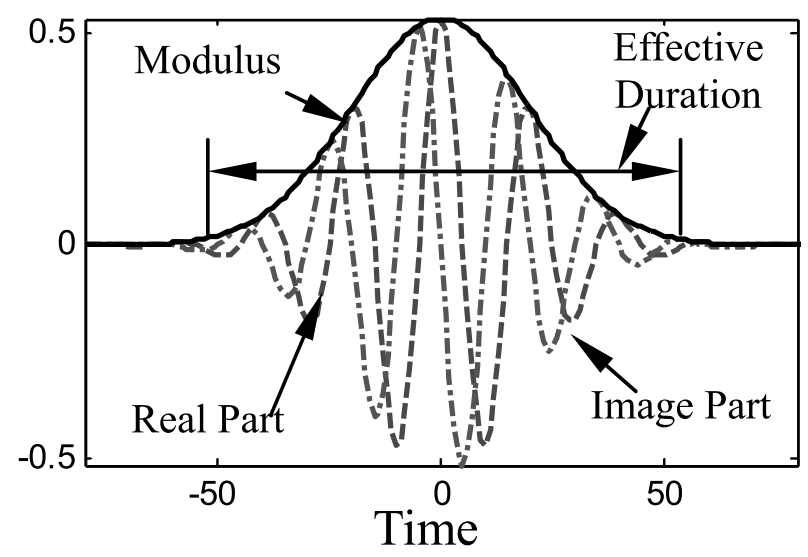

Fig. 8. A Morlet wavelet function $(\sigma=2$; Normalized frequency $=0.05 \mathrm{~Hz})$ Time / Sampling points.

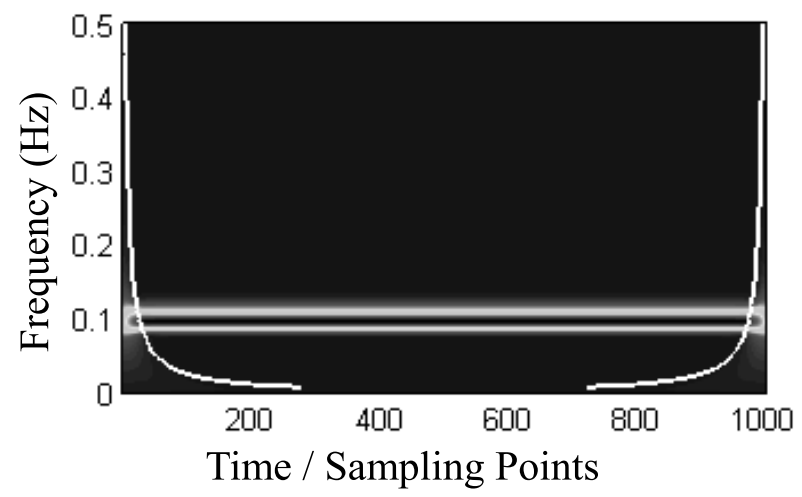

Fig. 9. The scalogram and the border distortion ranges.

$$
\Delta \psi_{1,0}=\left(\int_{-\infty}^{\infty} t^{2}\left|\psi_{1,0}(t)\right|^{2} d t\right)^{1 / 2}
$$

And according to the wavelet theory, the resolution window length of the wavelet function dilated with scale $a$ can then be calculates as

$$
\Delta \psi_{a, 0}=a \Delta \psi_{1,0}
$$

Therefore, at scale $a$, the border distortion range can be determined as

$$
R_{E E}(a)=a \lambda \Delta \psi_{1,0}
$$

where $\lambda$ is the proportionality factor which was suggested to be 3.0 in Ref [42]. Equation (14) clearly indicates that the border distortion effect range is wider at the low frequency region (big scale) than at the high frequency region (small scale).

Equation (14) provides a theoretical way to analyze the border distortion effect range, which, however, can be determined by an alternative method that can be conveniently conducted in practice simply using the effective duration of the wavelet function. As shown in Fig. 8, outside the effective duration, the modulus of the wavelet function is quite small and so the signal fraction outside this duration can only make insignificant contribution to the transform result and so can be neglected. The effective duration can be defined as the range in which the modulus of the wavelet function is bigger than a constant and the constant is proportional by factor $k$ ( $>1$ and suggested to be 5) to the maximum of the modulus of the wavelet function. And the border distortion effect range is half of the effective duration. By using this method, the border distortion effect ranges for the scalogram in Fig. 2 are determined and are shown in Fig. 9, in which the zones below the white lines are the border distortion effect ranges. 


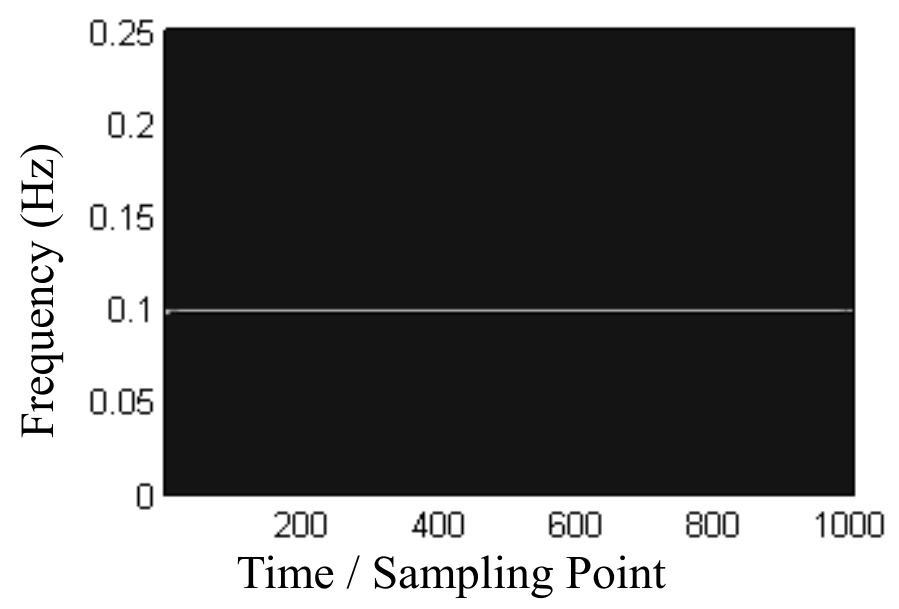

Fig. 10. The improved reassigned scalogram.

\subsection{Border distortion improvement methods}

In Section 3.1, the reasons for the occurrences of the three border distortion phenomena in the conventional and reassigned scalogram are investigated from the band-pass filter point of view. The understanding of the reasons for the border distortions allows designing effective methods to refine the scalograms and to alleviate the influences introduced by the border distortions.

To eliminate the ripple phenomenon from the scalogram at both the edges, it can resort to the priori knowledge about the wavelet function and the inspected signal themselves. First, the essential frequency components of the incomplete signal fraction would be bigger than the frequency components caused by the step components in amplitude. Second, the frequency pass-band of the wavelet function dilated by scale $a$ is always available and is denoted as $F P B(a)$. Based on the two facts, for the scalogram at point $(a, \underline{t})$ which is among the border distortion ranges, the following method is developed to eliminate the ripple:

1. Calculate the spectrum of the incomplete signal fraction at $t$, defined as $\underline{X}(j \omega)$;

2. Find the essential frequency components by a threshold which is defined as $\max (|X(j \omega)|) / \gamma$ where $\gamma$ is proportional factor. The components in $\underline{X}(j \omega)$ whose absolute amplitudes are bigger than the defined threshold are considered as the essential frequency components of the incomplete signal fraction;

3. Refine the scalogram. If there are some essential frequency components among pass-band $F P B(a)$, then keep the value at point $(a, \underline{t})$, otherwise, set the value at this point to zero.

To amend the results of the wavelet transform at the border distortion ranges so that the results can represent the energy distribution of the inspected signal even on the two edges, the equal-window-area method is recommended. This method is based on a fact that on the edges the value of wavelet transform is usually proportional to the area of the window. Therefore, the values of the wavelet transform can be amended as

$$
\bar{W}_{x}(a, b ; \psi)=\varsigma(a, b) W_{x}(a, b ; \psi)
$$

where $\varsigma(a, b)$ is the area ratio of the complete wavelet window to the wavelet window used at point $(a, b)$. Obviously, $\varsigma(a, b)$ is bigger than 1 on the two edges and is equal to 1 at the middle part.

As the fork shape on the two edges for the reassigned scalogram are mainly because of the inappropriate shift in time direction, a simple way to avoid this border distortion is then not to conduct the reassignment operation in time direction to the scalogram at the border distortion ranges. Here the reassigned scalogram in the border distortion ranges is defined as

$$
R S G_{x}(\hat{a}, b ; \psi)=\iint(\hat{a} / a)^{2} \overline{S G}_{x}(a, b ; \psi) \delta\left(\hat{a}-a^{\prime}(a, b)\right) d a d b
$$




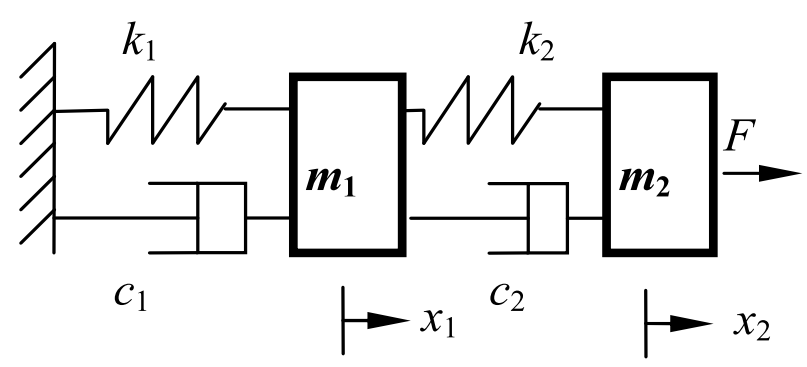

Fig. 11. A 2-DOF linear system.

where $\overline{S G}_{x}(a, b ; \psi)$ is the conventional scalogram after the border distortion cancellation. The improved version of the reassigned scalogram in Fig. 3 is shown in Fig. 10, which clearly indicates that the fork shapes disappear from the reassigned scalogram.

\section{The application for modal parameter estimation}

To be honest, the negative influences of the border distortions are not very significant for most practical applications, especially where the inspected objects are long signals in whose time frequency planes the effect ranges of the border distortion will only be a small proportion. However, when applying the wavelet transform for the modal parameter identification in the case where only short test data are available, the border distortion then will significantly affect the identification results, and therefore have draw particular attentions from the researchers in the system identification area, and so most discussions about the border distortion have appeared in the literatures about the modal parameter identification.

The powerful capability of the wavelet transform in system identification mainly lies in two facts $[25,27,29]: 1)$ by mapping the impulse response of the system to be identified, the wavelet transform can decouple the modes embedded in the impulse response in the time scale plane, and so the decoupled modes can be analyzed separately; 2) the ridge and skeleton of wavelet transform are of excellent capability in tracing the instantaneous characteristics of each mode. For the $i$-th mode, the impulse response can be expressed as the following form

$$
x_{i}(t)=A_{i} e^{-\xi_{i} \omega_{i} t} \sin \left(\sqrt{1-\xi_{i}^{2}} \omega_{i} t+\theta_{i}\right)
$$

where $\omega_{i}$ is the $i$-th modal frequency, $A_{i}$ is the $i$-th residue magnitude and $\xi_{i}$ is the $i$-th modal damping ratio, in the time scale plane the wavelet transform results along the ridge which is corresponding to the $i$-th mode will satisfy the following relationship

$$
\ln \left(\left|W_{x_{i}}\left(a_{i}(b), b ; \psi\right)\right|\right)-\delta \approx-\xi_{i} \sqrt{1-\xi_{i}^{2}} \omega_{i} b+\ln \left(A_{i}\right)
$$

where $\delta$ is a constant, $a_{i}(b)$ are the scale parameters along the ridge, in the linear system case, $a_{i}(b)$ would be the same and can be simply denoted as $a_{i}$. Moreover, usually, in practice $\xi_{i}^{2}<<1$, then Eq. (18) can be simplified as

$$
\ln \left(\left|W_{x_{i}}\left(a_{i}, b ; \psi\right)\right|\right)-\delta \approx-\xi_{i} \omega_{i} b+\ln \left(A_{i}\right)
$$

Equation (19) provides a convenient way to estimate the modal damping ratio $\xi_{i}$ simply by estimating the slope rate of $\ln \left(\left|W_{x_{i}}\left(a_{i}, b ; \psi\right)\right|\right)$ using the well-established least square method. In addition, the modal frequency $\omega_{i}$ can be estimated as $a_{i}=\omega_{0} / \omega_{i}$ when the Morlet function is used. If the scalogram rather than the wavelet transform result is used, then Eq. (19) needs to be modified as

$$
\frac{1}{2} \ln \left(\left|S G_{x_{i}}\left(a_{i}, b ; \psi\right)\right|\right)-\delta \approx-\xi_{i} \omega_{i} b+\ln \left(A_{i}\right)
$$

In this section, both the scalogram and reassigned scalogram will be applied to estimate the modal parameters for a 2-DOF linear system under the conditions of with and without measure noise. The comparisons will be carried out 
Table 1

The theoretical modal parameters

\begin{tabular}{ccc}
\hline Mode Number & Modal Frequency $(\mathrm{rad} / \mathrm{s})$ & Modal Damping $\left(\times 10^{-2}\right)$ \\
\hline Mode 1 & 38.8322 & 0.1942 \\
Mode 2 & 101.6641 & 0.5083 \\
\hline
\end{tabular}

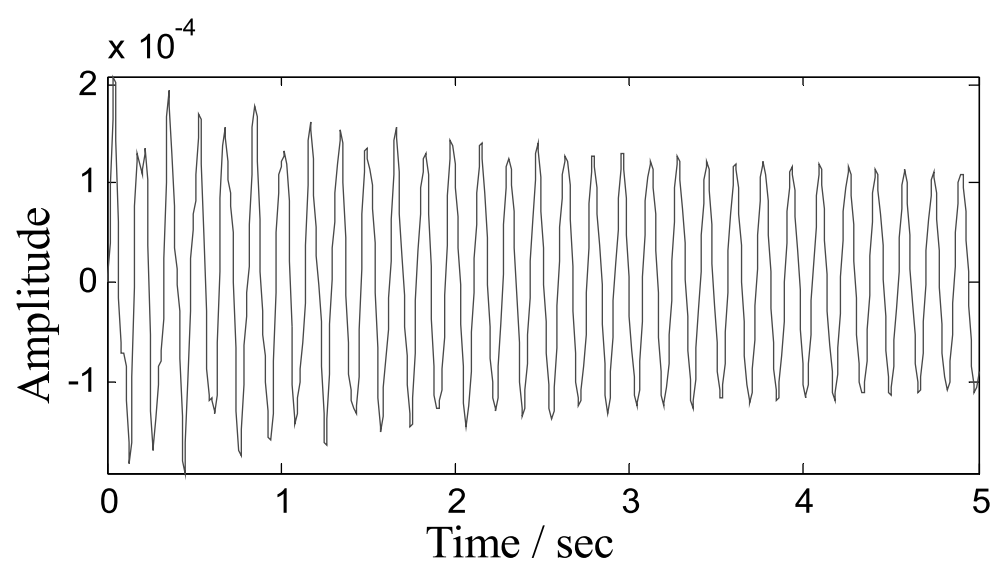

Fig. 12. The time domain waveform.

between the results obtained using the usual scalograms and the scalograms with the border distortion improvements. Figure 11 shows the considered 2-DOF linear system whose motion is governed by Eq. (21).

$$
M \ddot{x}+C \dot{x}+K x=F(t)
$$

where

$$
M=\left[\begin{array}{cc}
m_{1} & 0 \\
0 & m_{2}
\end{array}\right], \quad C=\left[\begin{array}{cc}
c_{1}+c_{2}-c_{2} \\
-c_{2} & c_{2}
\end{array}\right], \quad K=\left[\begin{array}{cc}
k_{1}+k_{2}-k_{2} \\
-k_{2} & k_{2}
\end{array}\right]
$$

are the system mass, damping and stiffness matrix respectively. $x=\left(x_{1}, x_{2}\right)^{\prime}$ is the displacement vector, and $F$ is the external impulse force imposed on $m_{2}$. In the present numerical study, the damping is assumed to be proportional to the stiffness, e.g., $C=\mu K$. The values of the system parameters are taken as

$$
m_{1}=m_{2}=1, k_{1}=k_{2}=3.9748 \times 10^{3}, \mu=0.0001
$$

It is easy to calculate the theoretical modal parameters for this 2-DOF system and the results are given in Table 1.

\subsection{Case 1: Noise Free}

In this case, the impulse force are used with strength $=10 \mathrm{~N}$. The impulse response is obtained using the fourthorder Runge-Kutta method with sampling frequency $=120 \mathrm{~Hz}$. The time domain waveform and the conventional scalogram and the reassigned scalogram of the response of mass 1 are shown in Figs 12, 13, 14 respectively. In the conventional scalogram, the ridges are marked with two white color lines which are corresponding to the two modes. The ridges are not marked out in the reassigned scalogram for the clear of picture. Figure 15 shows the logarithmical values of the scalograms along the ridges for both the usual scalograms and the scalograms after border distortion improvements. From Figs 13 and 14, the modal frequencies of mode 1 and mode 2 can be easily obtained, and then from Fig. 15 the modal damping ratios can be estimated for mode 1 and mode 2 using the least square method based on Eq. (20). When using ridges extracted from the usual scalograms, only the middle parts are used. The final results are listed in Table 2. The results clearly indicate that there are no differences between the estimated results respectively provided by the usual scalograms and the scalograms with the border distortion improvements. The result differences between the conventional scalogram method and the reassigned scalogram method are mainly caused the frequency discrete in the frequency axis. 


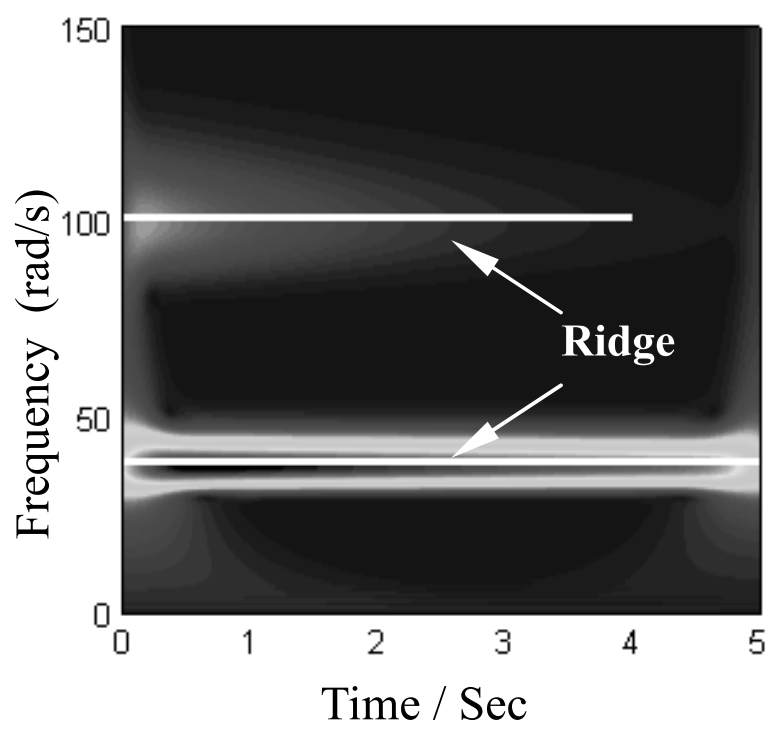

Fig. 13. The scalogram and the ridge.

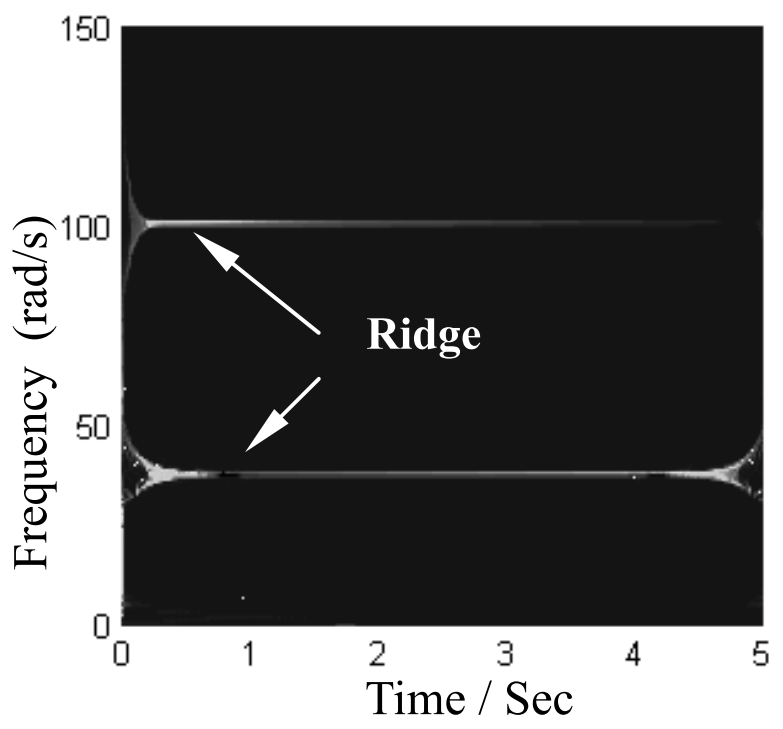

Fig. 14. The reassigned scalogram and the ridge.

\subsection{Case 2: With noise}

In this case, the impulse response signals are supposed to be contaminated by white noise and the maximum amplitude of the noise is assumed to be $20 \%$ of the maximum amplitude of the measured response. The noise contaminated signal is shown in Fig. 16. The conventional scalogram and the reassigned are shown in Figs 17 and 18. In the conventional scalogram the ridges associated the two modes are marked with two white color lines. It can be seen that the effective ridge for mode 2 is very short as the amplitude of mode 2 self is very weak and the associated frequency component is not clearly even before $1.5 \mathrm{sec}$ and is nearly masked by the noise component after $1.5 \mathrm{sec}$. This raises the challenge to estimate the modal parameters for mode 2 . On the contrary, the frequency 
Table 2

The estimated modal parameters

\begin{tabular}{cccccc}
\hline Method & \multicolumn{2}{c}{ Mode 1 } & & \multicolumn{2}{c}{ Mode 2 } \\
\cline { 2 - 3 } \cline { 5 - 6 } & $\begin{array}{c}\text { Modal frequency } \\
(\mathrm{rad} / \mathrm{s})\end{array}$ & $\begin{array}{c}\text { Modal damping } \\
\left(\times 10^{-2}\right)\end{array}$ & & $\begin{array}{c}\text { Modal frequency } \\
(\mathrm{rad} / \mathrm{s})\end{array}$ & $\begin{array}{c}\text { Modal damping } \\
\left(\times 10^{-2}\right)\end{array}$ \\
\hline 1 & 39.0357 & 0.1951 & & 101.6400 & 0.5100 \\
2 & 39.0357 & 0.1951 & & 101.6400 & 0.5100 \\
3 & 38.2992 & 0.1989 & & 100.9035 & 0.5137 \\
4 & 38.2992 & 0.1989 & & 100.9035 & 0.5137 \\
\hline
\end{tabular}

( ${ }^{*}$ Method 1 - Usual Scalogram; Method 2 - Improved Scalogram; Method 3 - Usual Reassigned Scalogram; Method 4 - Improved Reassigned Scalogram).
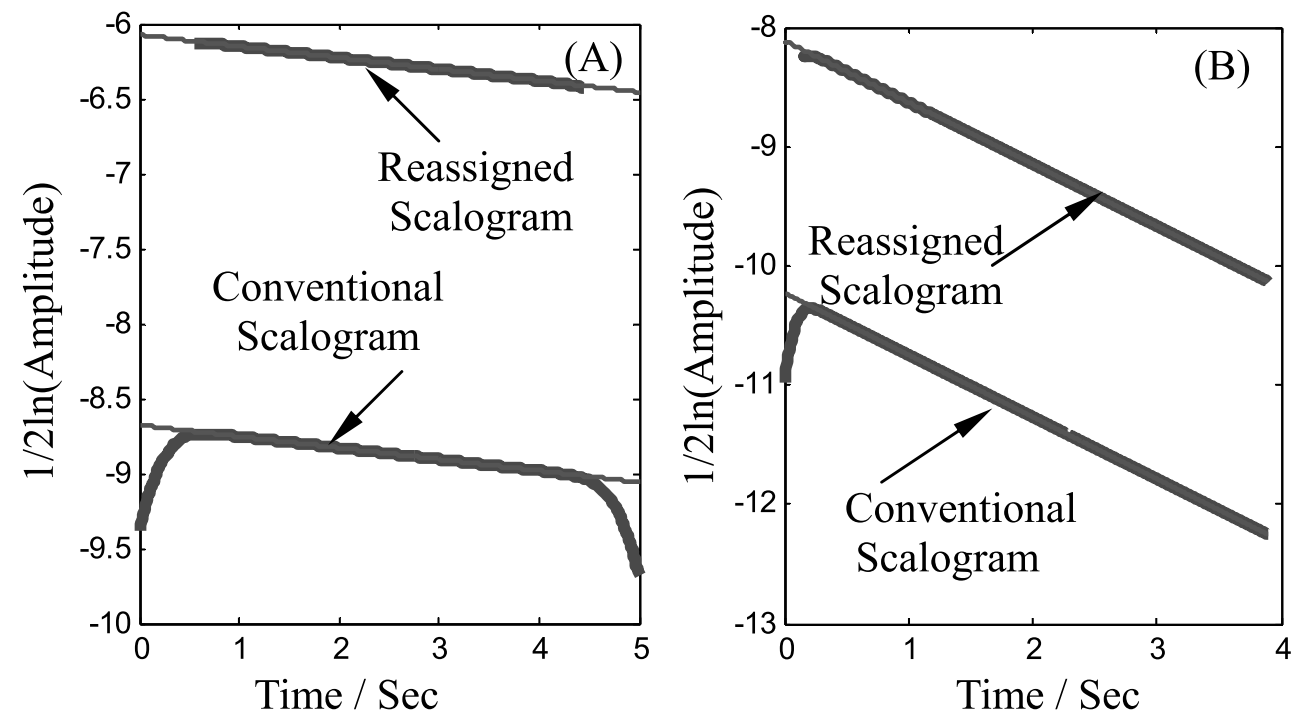

Fig. 15. The logarithmical values of the scalograms along the ridges [(A) - Mode 1; (B) - Mode 2].

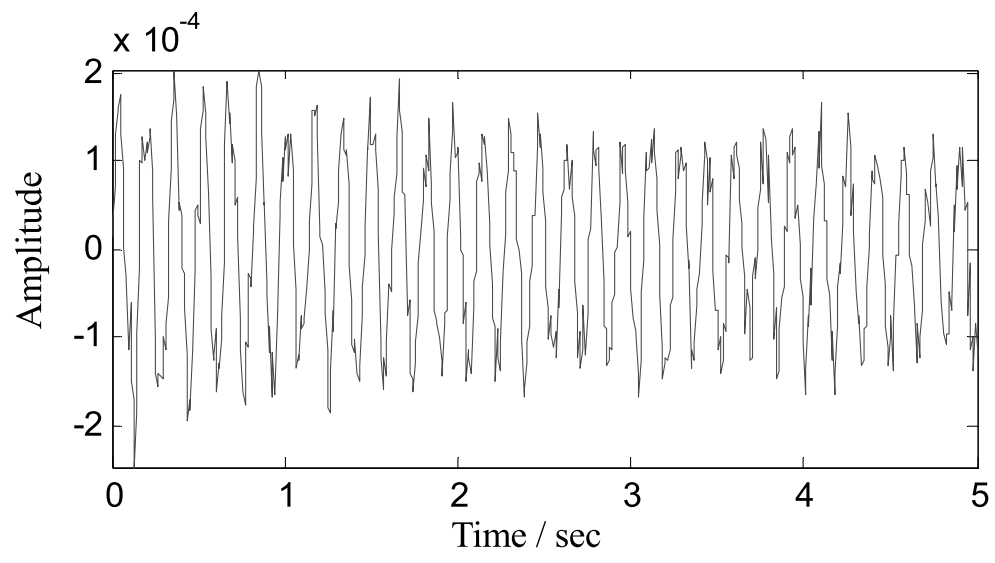

Fig. 16. The signal contaminated by noise.

component associated to mode 2 is relatively clearer in the reassigned scalogram as the better energy concentration of the reassigned scalogram can improve the signal noise ratio (SNR).

Figure 19 shows the logarithmical values of the scalograms along the ridges for both the usual scalograms and the scalograms after border distortion improvements, from which the modal parameters are estimated. The final 


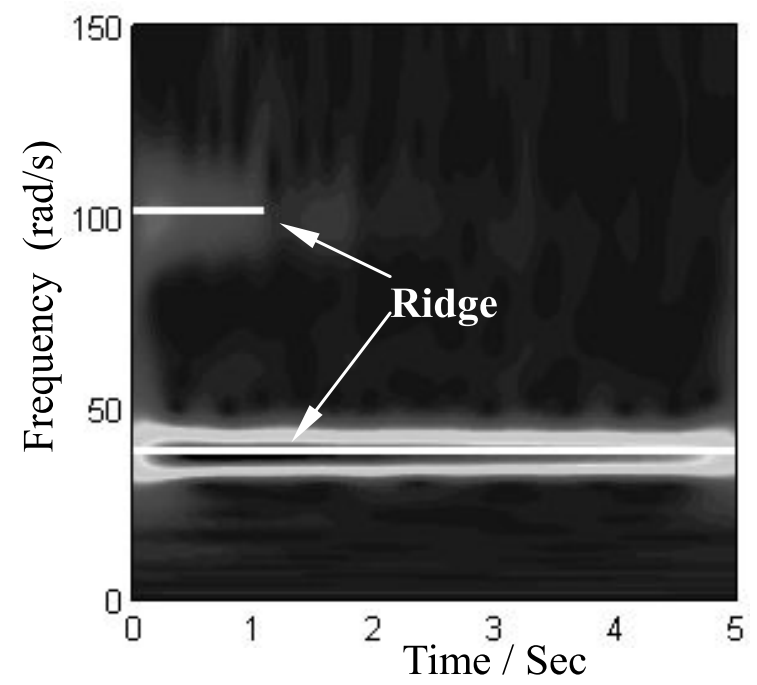

Fig. 17. The scalogram and the ridge.

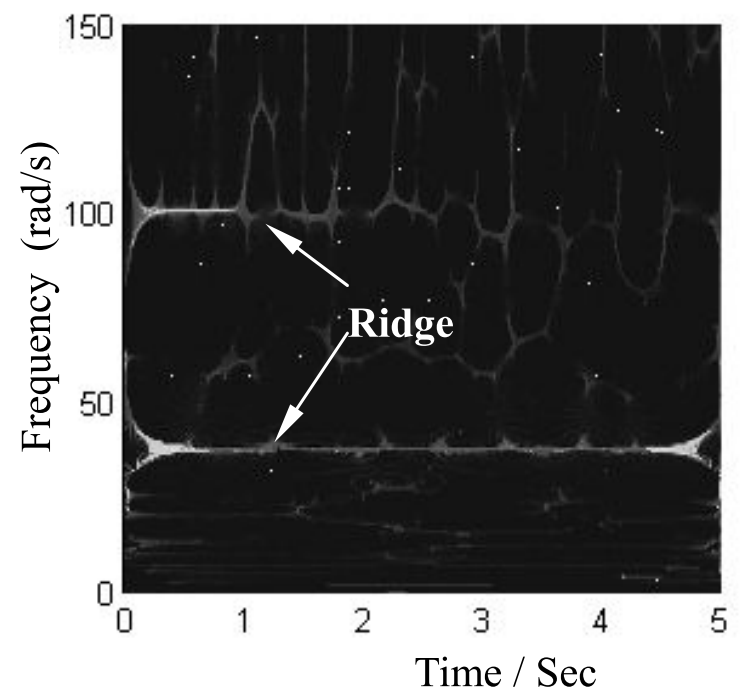

Fig. 18. The reassigned scalogram and the ridge.

estimation results are given in Table 3. It can be seen that there are no significant differences between the results about the modal parameters of mode 1 , and all estimation results are close to the real values. It is because the amplitude of mode 1 is big enough and the associated frequency component is quite strong, consequently, the SNR is relatively high and, therefore, the negative effects of the noise on the estimation results are little. However, the influences of the noise on the estimation results of the modal damping of mode 2 are somewhat significant as the amplitude of this mode is not strong enough to resist the effects of the noise component. Clearly, the results estimated by using the scalograms with border distortion improvement are much better than those using the usual scalograms. It is not surprising as mode 2 is actually stronger at the border distortion range than at the middle part and thus the SNR is high. Taking into account of the frequency components at the border distortion range is therefore able to improve the estimation results. The results in Table 3 also indicate that the reassigned scalograms can do better than the conventional scalograms. As aforementioned, it is because that the reassigned scalograms possess better energy concentration and so the SNR is high. 
Table 3

The estimated modal parameters

\begin{tabular}{cccccc}
\hline Method & \multicolumn{2}{c}{ Mode 1 } & & \multicolumn{2}{c}{ Mode 2 } \\
\cline { 2 - 3 } \cline { 5 - 6 } & $\begin{array}{c}\text { Modal frequency } \\
(\mathrm{rad} / \mathrm{s})\end{array}$ & $\begin{array}{c}\text { Modal damping } \\
\left(\times 10^{-2}\right)\end{array}$ & & $\begin{array}{c}\text { Modal frequency } \\
(\mathrm{rad} / \mathrm{s})\end{array}$ & $\begin{array}{c}\text { Modal damping } \\
\left(\times 10^{-2}\right)\end{array}$ \\
\hline 1 & 39.0357 & 0.1878 & & 101.6400 & 0.3710 \\
2 & 39.0357 & 0.1893 & & 101.6400 & 0.4351 \\
3 & 39.0357 & 0.1880 & & 100.9035 & 0.4557 \\
4 & 39.0357 & 0.1913 & & 100.9035 & 0.4912 \\
\hline
\end{tabular}

( ${ }^{*}$ Method 1 - Usual Scalogram; Method 2 - Improved Scalogram; Method 3 - Usual Reassigned Scalogram; Method 4 - Improved Reassigned Scalogram).
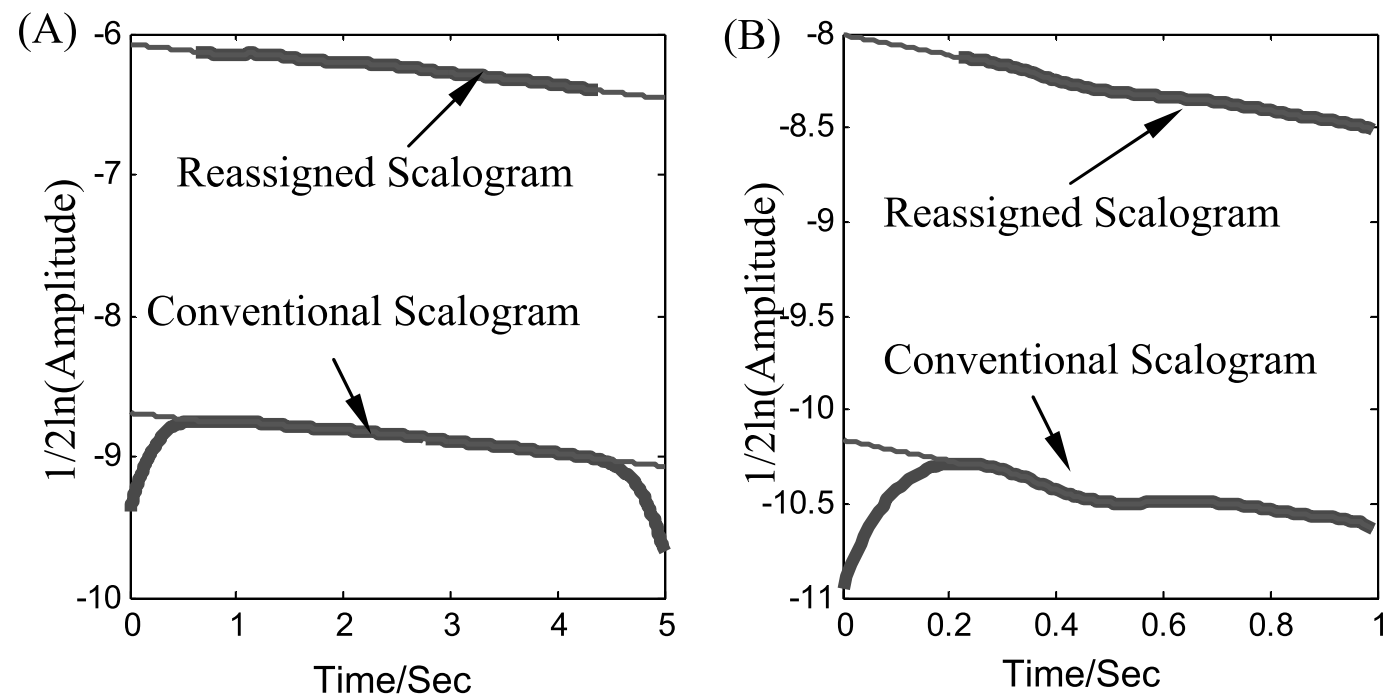

Fig. 19. The logarithmical values of the scalograms along the ridges [(A)-Mode 1; (B) - Mode 2].

\section{Conclusions}

In the present work, from the band-pass filter point of view, the causes for the border distortions have been investigated for the conventional scalogram and for the reassigned scalogram. New methods were then put forward to reduce the influences introduced by the border distortions to both the scalograms. All the scalograms with and without border distortion improvements have been applied to estimate the modal parameters for a 2-DOF linear system. The estimation results indicate that all the scalograms can accurately estimate the modal parameters, provided that the measured response signals are not contaminated by noise. In the presence of noise, for the modes whose associated frequency components are not strong enough, the reassigned scalogram can perform better than the conventional scalogram in estimating the modal parameters. Moreover, for the modes whose effective durations are not long enough, the frequency components at the border distortion ranges are of great significance to the estimation results, and the border distortion improvement method will then make sense in the application of the wavelet transform for the modal parameter estimation.

\section{Acknowledgements}

The authors gratefully acknowledge the supports of the Natural Science Foundation of China (No. 10902068 and No. 10732060), the NCET in University of China (No. NCET-10-0548), and the 863 Program (No. 50821003), for this work. 


\section{References}

[1] I. Daubechies, The wavelet transform, time-frequency localization and signal analysis, IEEE Transactions on Information Theory 36 (1990), 961-1005.

[2] C. Torrence and G.P. Compo, A Practical Guide to Wavelet Analysis, Bulletin of the American Meteorological Society 79 (1998), 61-78.

[3] Q. Sun and Y. Tang, Singularity analysis using continuous wavelet transform for bearing fault diagnosis, Mechanical Systems and Signal Processing 16 (2002), 1025-1041.

[4] Z.K. Peng, F.L. Chu and P.W. Tse, Singularity analysis of the vibration signals by means of wavelet modulus maximal method, Mechanical Systems and Signal Processing 21 (2007), 780-794.

[5] Z. Peng, Y. He, Z. Chen and F. Chu, Identification of the shaft orbit for rotating machines using wavelet modulus maxima, Mechanical Systems and Signal Processing 16 (2002), 623-635.

[6] J.C. Hong, Y.Y. Kim, H.C. Lee and Y.W. Lee, Damage detection using the Lipschitz exponent estimated by the wavelet transform: applications to vibration modes of a beam, International Journal of Solids and Structures 39 (2002), 1803-1816.

[7] Y.Y. Kim, J.C. Hong and N.Y. Lee, Frequency response function estimation via a robust wavelet de-noising method, Journal of Sound and Vibration 244 (2001), 635-649.

[8] I.S. Bozchalooi and M. Liang, A smoothness index-guided approach to wavelet parameter selection in signal de-noising and fault detection, Journal of Sound and Vibration 308 (2007), 246-267.

[9] H.X. Chen, Patrick S.K. Chua and G.H. Lim, Adaptive wavelet transform for vibration signal modelling and application in fault diagnosis of water hydraulic motor, Mechanical Systems and Signal Processing 20 (2006), 2022-2045.

[10] E.B. Halim, M.A.A.S. Choudhury, S.L. Shah and M.J. Zuo, Time domain averaging across all scales: A novel method for detection of gearbox faults, Mechanical Systems and Signal Processing 22 (2008), 261-278.

[11] C. Kar and A.R. Mohanty, Vibration and current transient monitoring for gearbox fault detection using multiresolution Fourier transform, Journal of Sound and Vibration (2007), doi:10.1016/j.jsv.2007.08.023.

[12] T.J. Johnson and D.E. Adams, Composite indices applied to vibration data in rolling tires to detect bead area damage, Mechanical Systems and Signal Processing 21 (2007), 2161-2184.

[13] H Ocak, K.A. Loparo and F.M. Discenzo, Online tracking of bearing wear using wavelet packet decomposition and probabilistic modeling: A method for bearing prognostics, Journal of Sound and Vibration 302 (2007), 951-961.

[14] O. Rioul and M. Vetterli, Wavelets and signal processing, IEEE Signal Processing Magazine 8 (2001), 14-38.

[15] http://en.wikipedia.org/wiki/Short-time_Fourier_transform.

[16] Z. Peng, F. Chu and Y. He, Vibration signal analysis and feature extraction based on reassigned wavelet scalogram, Journal of Sound and Vibration 253 (2002), 1087-1100.

[17] C Smith, C.M. Akujuobi, P. Hamory and K. Kloesel, An approach to vibration analysis using wavelets in an application of aircraft health monitoring, Mechanical Systems and Signal Processing 21 (2007), 1255-1272.

[18] Z. Peng, Y. He, Q. Lu and F. Chu, Feature extraction of the rubbing caused impacts rotor system by means of wavelet analysis, Journal of Sound and Vibration 259 (2003), 1000-1010.

[19] Y.P. Zhang, S.H. Huang, J.H. Hou, T. Shen and W. Liu, Continuous wavelet grey moment approach for vibration analysis of rotating machinery, Mechanical Systems and Signal Processing 20 (2006), 1202-1220.

[20] C.S. Huang and W.C. Su, Identification of modal parameters of a time invariant linear system by continuous wavelet transformation, Mechanical Systems and Signal Processing 21 (2007), 1642-1664.

[21] S. Erlicher and P. Argoul, Modal identification of linear non-proportionally damped systems by wavelet transform, Mechanical Systems and Signal Processing 21 (2007), 1386-1421.

[22] T.-P. Le and P. Argoul, Continuous wavelet transform for modal identification using free decay response, Journal of Sound and Vibration 277 (2004), 73-100

[23] A. Chakraborty, B. Basu and M. Mitra, Identification of modal parameters of a mdof system by modified L-P wavelet packets, Journal of Sound and Vibration 295 (2006), 827-837.

[24] J. Slavc, I. Simonovski and M. Boltezar, Damping identification using a continuous wavelet transform: application to real data, Journal of Sound and Vibration 262 (2003), 291-307.

[25] M. Boltezar and J. Slavic, Enhancements to the continuous wavelet transform for damping identifications on short signals, Mechanical Systems and Signal Processing 18 (2004), 1065-1076.

[26] B.F. Yan, A. Miyamoto and E. Brühwiler, Wavelet transform-based modal parameter identification considering uncertainty, Journal of Sound and Vibration 291 (2006), 285-301.

[27] J. Lardies and S. Gouttebroze, Identification of modal parameters using the wavelet transform International Journal of Mechanical Sciences 44 (2002), 2263-2283.

[28] R. Ghanem and F. Romeo, A wavelet-based approach for model and parameter identification of non-linear systems, International Journal of Non-Linear Mechanics 36 (2001), 835-859.

[29] W.J. Staszewski, Identification of damping in MDOF systems using time-scale decomposition, Journal of Sound and Vibration 203 (1997), 283-305.

[30] W.J. Staszewski, Identification of non-linear systems using multi-scale ridges and skeletons of the wavelet transform, Journal of Sound and Vibration 214 (1998), 639-658.

[31] A.N. Robertson, K.C. Park and K.F. Alvin, Identification of structural dynamics models using wavelet-generated impulse response data, Journal of Vibration and acoustics 120 (1998), 261-266.

[32] M. Ruzzene, A. Fasana, L. Garibaldi and B. Piombo, Natural frequencies and dampings identification using wavelet transform: application to real data, Mechanical Systems and Signal Processing 11 (1997), 207-218. 
[33] D.E. Newland, Ridge and phase identification in the frequency analysis of transient signals by harmonic wavelets, Journal of Vibration and Acoustics 121 (1999), 149-155.

[34] Z.K. Peng and F.L. Chu, Application of the wavelet transform in machine condition monitoring and fault diagnostics: a review with bibliography, Mechanical Systems and Signal Processing 18 (2004), 199-221.

[35] F. Auger and P. Flandrin, Improving the readability of time-frequency and time-scale representations by the reassignment method, IEEE Transactions on Signal Processing 43 (1995), 1068-1089.

[36] P.W. Tse, W.X. Yang and H.Y. Tam, Machine fault diagnosis through an effective exact wavelet analysis, Journal of Sound and Vibration 277 (2004), 1005-1024.

[37] W.-X. Yang, A natural way for improving the accuracy of the continuous wavelet transforms, Journal of Sound and Vibration 306 (2007), 928-939.

[38] K. Kodera, R. Gendrin and C. Villedary, Analysis of time-varying signals with small BT values, IEEE Transactions on Acoustics, Speech and Signal Processing ASSP-26 (1978), 64-76.

[39] Z.K. Peng, F.L. Chu and P.W. Tse, Detection of the rubbing-caused impacts for rotor-stator fault diagnosis using reassigned scalogram, Mechanical Systems and Signal Processing 19 (2005), 391-409.

[40] S. Su and R. Du, Signature analysis of mechanical watch movements, Mechanical Systems and Signal Processing 21 (2007), 3189-3200.

[41] I. Simonovski and M. Boltezar, The norms and variances of the Gabor, Morlet and general harmonic wavelet functions, Journal of Sound and Vibration 264 (2003), 545-557.

[42] T. Kijewski and A. Kareem, On the presence of end effects and their melioration in wavelet-based analysis, Journal of Sound and Vibration 256 (2002), 980-988. 

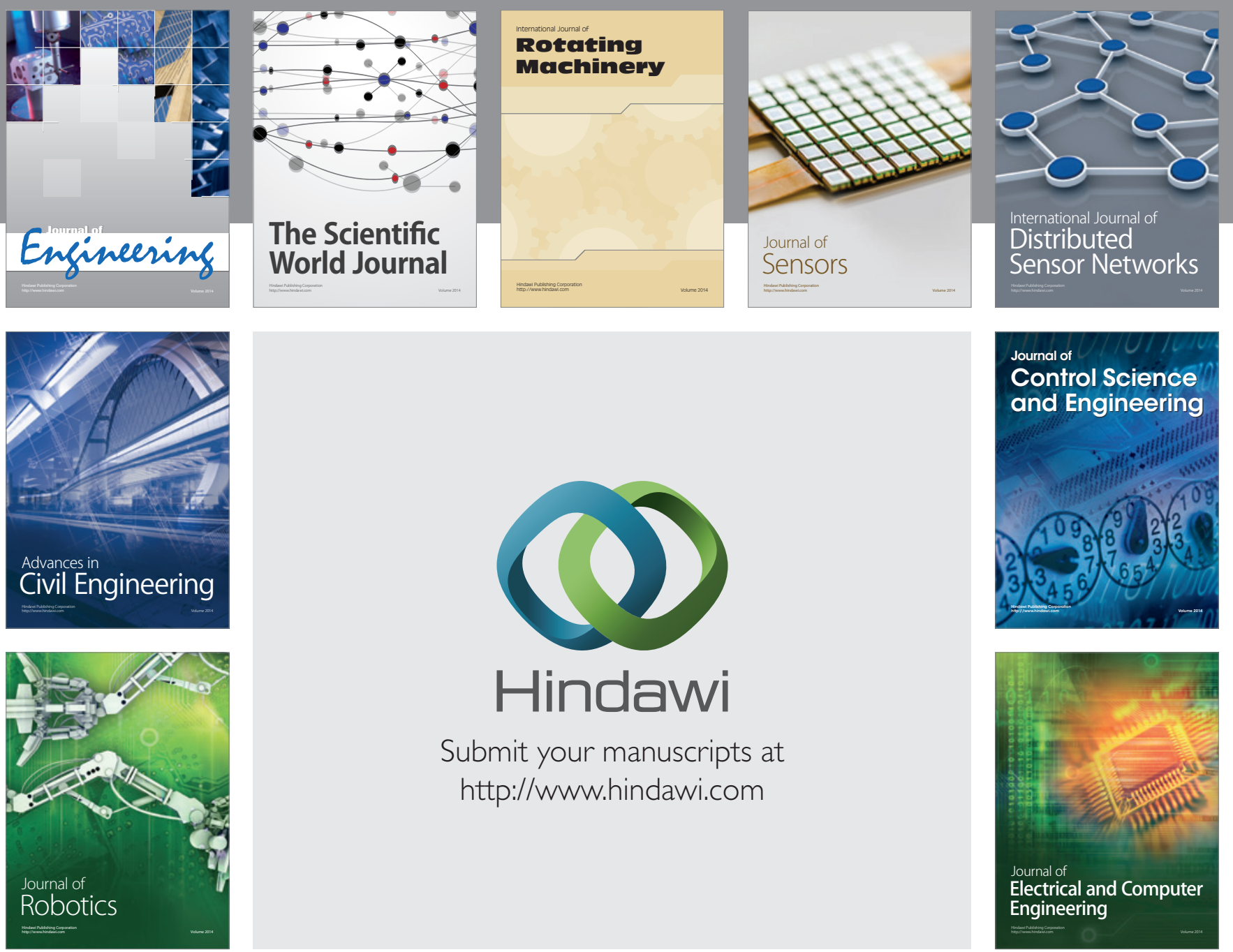

Submit your manuscripts at

http://www.hindawi.com
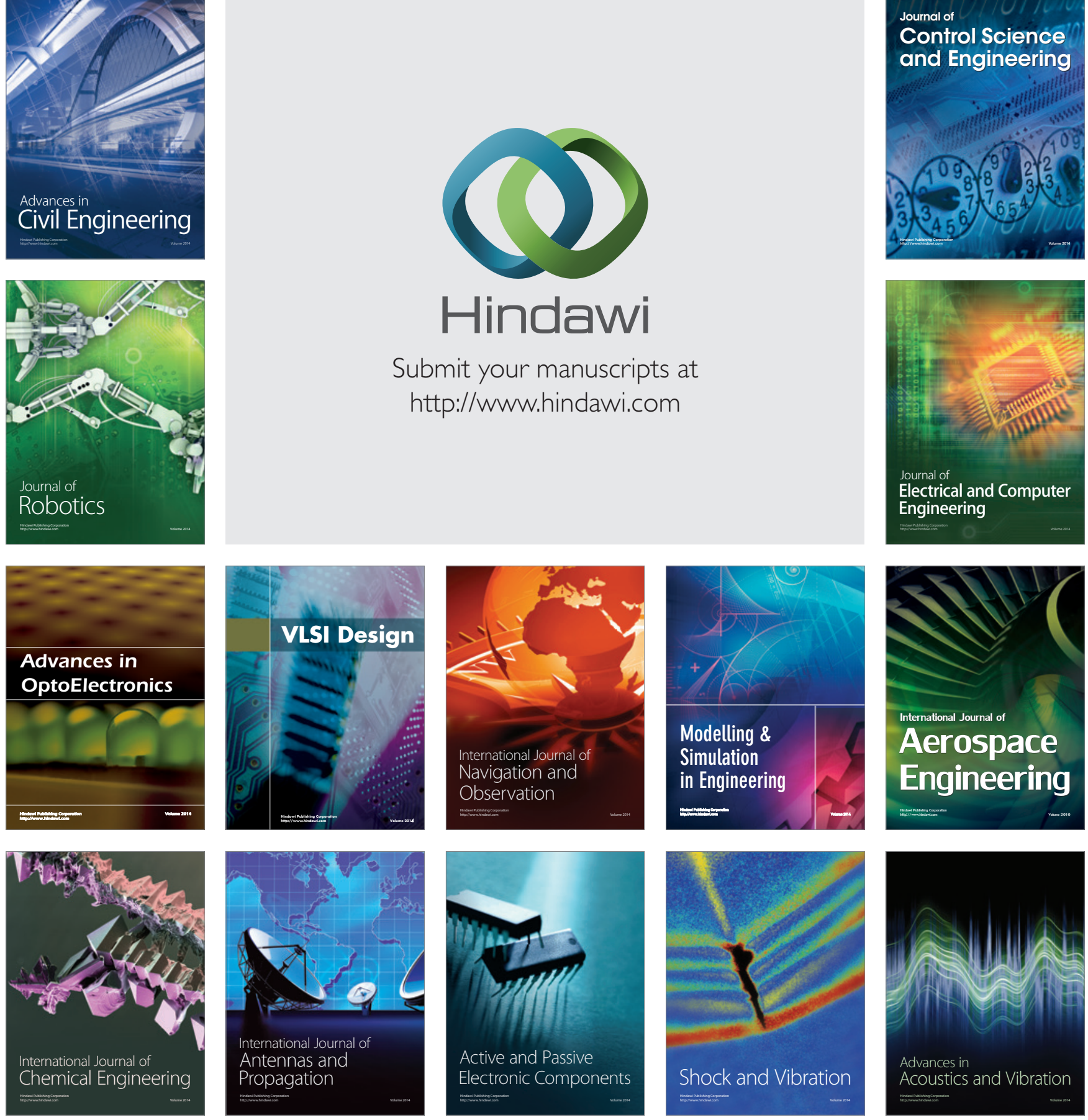